\title{
Erzählen im Journalismus
}

\author{
Matías Martínez
}

\section{Erzählen im Journalismus: Bestimmung des Gegenstandsbereichs}

Gibt es bestimmte soziale Funktionen des Journalismus, aus denen man Merkmale journalistischer Wirklichkeitserzählungen ableiten kann? Niklas Luhmann hat vorgeschlagen, den Journalismus als Teil der Massenmedien zu verstehen, die ein eigenständiges soziales System innerhalb unserer funktional differenzierten Gesellschaft bildeten. Der spezifische Code, der das System der Massenmedien von seiner Umwelt abgrenze, sei die Unterscheidung zwischen Information und Nichtinformation. Die Massenmedien, so Luhmann, stünden unter einem dauernden Erneuerungsdruck wegen der »ständigen Deaktualisierung von Information « ${ }^{1} »$ Informationen lassen sich nicht wiederholen; sie werden, sobald sie Ereignis werden, zur Nichtinformation. Eine Nachricht, die ein zweites Mal gebracht wird, behält zwar ihren Sinn, verliert aber ihren Informationswert ${ }^{2}{ }^{2} \mathrm{Im}$ Einzelnen ordnet Luhmann den Massenmedien (nicht im Sinne einer »geschlossenen Typologie«, sondern »rein induktiv«) drei »Programmbereiche« zu: »Werbung «, "Unterhaltung « und "Nachrichten und Berichte«. ${ }^{3}$ Bernd Blöbaum beschreibt den für »Nachrichten und Berichte« zuständigen Journalismus nicht als Teil des sozialen Systems der Massenmedien, sondern als ein eigenständiges soziales System, dem, so wie den Massenmedien insgesamt bei Luhmann, als primäre Funktion die »aktuelle Selektion und Vermittlung von Informationen« zukomme. ${ }^{4}$ In beiden Fällen wird der Journalismus jedenfalls systemtheoretisch durch die Leitdifferenz zwischen Information und Nichtinformation bestimmt. Obwohl selbstverständlich nicht nur im Journalismus, sondern auch in der Werbung und in der Unterhaltung erzählt wird, geht es im vorliegenden Beitrag, der engeren Bestimmung Blöbaums folgend, um Formen und Funktionen des Erzählens im Journalismus, und zwar um das rein sprachliche Erzählen im Printjournalismus von Zeitungen und Zeitschriften.

Was Luhmann als den ,Informationswert einer Nachricht bezeichnet, wird in der publizistischen Theorie des Nachrichtenwerts in ein Bündel unterschiedli-

1 Niklas Luhmann: Die Realität der Massenmedien, 2., erw. Aufl., Opladen 1996, S. 43, vgl. S. 36. Vgl. Matthias Kohring: "Journalismus als soziales System: Grundlagen eincr systemorienticrten Journalismustheorice, in: Martin Löffelholz (Hg.): Theorien des Journalismus. Ein diskerrsives Handbuch, 2., vollst. überarb. u. crw. Aufl., Wiesbaden 2004, S. 185-200.

2 Luhmann (Anm. 1), S. 41

3 Juhmann (Anm. 1), S. 51.

4 Bernd Blöbaum: Journalismns als soĩales System. Gescbicbte, Ausdifferenqiernng und Verselbständigung, Opladen 1994, S. 261. 
cher Faktoren zerlegt. ${ }^{5}$ Die Redaktionen der Zeitungen und Zeitschriften melden nur einen kleinen Teil der Informationen (bis zu 10 Prozent) weiter, die sie täglich durch die Nachrichtenagenturen erreichen - und auch diese sind bereits das Ergebnis einer sehr starken Selektion aller Meldungen, die täglich anfallen. Die Auswahl der Meldungen erfolgt nach ihrem Nachrichtenwert, der von den inhaltlichen Merkmalen (Nachrichtenfaktoren ) einer Meldung bestimmt wird. Zu den Nachrichtenfaktoren gehören u.a. das Ausmaß und die Konsequenzen des gemeldeten Ereignisses, seine Aktualität, räumliche und zeitliche Nähe, die Auswirkungen auf die eigene (nationale, regionale oder lokale) Gemeinschaft, die Prominenz der beteiligten Personen sowie ein sehr heterogenes Faktorenbündel, das unter human interest zusammengefasst wird: Skurrilität (HHund beißt Mann ist keine Nachricht, wohl aber sMann beißt Hund), Kampf, Konflikt, Sex, Liebe, Romantik, Humor, Abenteuer, Spannung, Tiere, Alter, Tragödie.

\section{Formen und Geltungsansprüche journalistischen Erzählens}

Journalisten bedienen sich unterschiedlicher Textsorten. ${ }^{6}$ Es gibt nicht-narrative Formen wie den Essay, das Feature, den Kommentar, die Glosse, die Kulturkritik, die Polemik oder das Interview. Zu den narrativen Formen gehören die Nachricht (oder Meldung), der Bericht und die Reportage. Aus diesen drei Gattungen bestehen die Wirklichkeitserzählungen im Feld des Printjournalismus.

\section{Nachribt}

Im Sinne einer bestimmten Form der journalistischen Darstellung (nicht im weiten Sinne von ,Information oder ,Mitteilung) bezeichnet man als Nachricht (oder auch: Meldung) einen kurzen Text, der in der Zeitung bis zu 25 Druckzeilen umfasst. Sie dient der knappen Informationsvermittlung und ist faktenorientiert. Jede Nachricht soll die sogenannten ,W-Fragen beantworten: Was? Wann? Wo? Wer? Wie? Warum?; außerdem wird oft die Herkunft der Information genannt: Welche Quelle? Der Kern der Information erscheint in kürzester Form bereits in der Überschrift und wird zu Beginn der Nachricht (je nach Gesamtlänge des Artikels im ersten Satz oder im ersten Absatz) zusammengefasst. Der Textaufbau wird deshalb in der Journalistik gern mit der Gestalt einer sumgekehrten Pyramider (inverted pyramid) verglichen: Der Textanfang (die Pyramidenspitze) ist sozusagen kompakter als das Ende (die Basis). Die Form des Nachrichtentextes

5 Zur Theoric des Nachrichtenwerts s. Joachim Friedrich Staab: „Entwicklungen der Nachrichtenwert-Theorie. Theoretische Konzepte und empirische Überprüfungen«, in: Jürgen Wilke (Hg.): Fortschritte der Publiqistikmissenschaft, Freiburg/München 1990, S. 161-172; Siegfried Weischenberg: Nachrichtenschreiben. Jommalistische Praxis zum Studiwm und Selbststudium, 2. Aufl., Opladen 1990, \$. 16-24.

6 Tïnc knappe Ubcrsicht gibt Erich Straßncr: "Zeitschriftenspe’ifische Präsentationsformen und Texttypen巛, in: Joachim-Felix Lconhard u.a. (Hg.): Medienwissenschaft. Ein Handbuch zutr Entwicklnng der Medien und Konmunikationsformen, 2. Tcilbd. (= Handbücher zur Sprach- und Kommunikationswissenschaft, Bd. 15.2), Berlin 2001, S. 1734-1739. 
wird hier also unmitrelbar durch die dominante Funktion des Journalismus (Information vs. Nichtinformation) geprägt.

Bericht

Der Bericht ist länger und inhaltlich umfassender als die Nachricht. Er gibt eine detailliertere, verlaufsorientierte, chronologisch-lineare Darstellung des Ereignisses und informiert nicht nur über Ort und Zeit und die beteiligten Personen, sondern auch über die Ursachen des Geschehens. Auch der Bericht soll nach dem Prinzip der umgekehrten Pyramide das Wichtigste an den Anfang stellen und die W-Fragen beantworten.

Reportage

Reportage ist der Sammelname für alle Großformen der Berichterstattung in den Printmedien. Anders als bei Nachrichten und Berichten, die in der Redaktion auf der Grundlage von Agenturmeldungen geschrieben werden, gehen der Reportage in der Regel eigenständige Recherchen des Reporters vor Ort voraus. Nicht alle Reportagen sind Erzählungen in dem Sinne, dass sie über ein Geschehen berichten würden - es gibt auch Reportagen über Personen, Zustände oder Hintergründe. Die Gestaltungsformen der Reportage sind variabler als bei den beiden kürzeren Arten journalistischer Wirklichkeitserzählungen. Reportagen lassen sich durch ihre Themen unterscheiden: Lokalreportagen, Sensationsreportagen, Sozialreportagen, Reportagen aus der Arbeitswelt, Enthüllungsreportagen, Kriegsreportagen, politische Reportagen, Gerichtsreportagen, Sportreportagen, Wissenschaftsreportagen, Reisereportagen. Allen diesen Reportagetypen ist gemeinsam, dass sie nicht auf eine neutrale Informationsvergabe zielen, sondern subjektiv getönte Darstellungen realer Ereignisse liefern. Sie wollen dem Leser nicht nur ein bestimmtes Ereignis, sondern auch das Erlebnis dieses Ereignisses vermitteln. Häufig geschieht das dadurch, dass der Reporter, als Stellvertreter des Lesers, aus der Perspektive eines teilnehmenden Beobachters erzählt.

Die moderne Reportage hat zahlreiche historische Vorläufer im Bereich des nichtliterarisch-faktualen und des literarisch-fiktionalen Erzählens. Zu ihren nichtliterarischen Quellen gehören, neben Selbstzeugnissen (Autobiographien, Memoiren, Konfessionen), Biographien, Briefen, Augenzeugenberichten (über Kriege, Naturkatastrophen usw.) und Parlamentsberichten, die Reiseerzählungen seit Herodots antiken Historien. ${ }^{7}$ Der Geltungsanspruch dieser Gattung sei am Beispiel von Johann Gottfried Seumes Spaqiergang nach Syrakus in Jabre 1802 (1803) illustriert. In der Vorrede schreibt Seume:

In Romancn hat man uns nun lange genug alte, nicht mehr gelcugnete Wahrheiten dichterisch eingekleidet, dargestellt und tausendmal wiederholt. Ich tadle dieses nicht, es ist det Anfang: aber immer nur Milchspeise für Kinder. Wir sollten doch endlich auch Männcr werden und beginnen, dic Sachen ernsthaft geschichtsmäßig zu nehmen, ohne Vorurteil und Groll, ohne I.eidenschaft und Selbstsucht. Örter, Personen, Namen, Umstände soll-

7 Vgl. Michael Haller: Die Reportage, 6., überarb. Aufl., Konstanz 2008, S. 17-59. 
ten immer bei den Tatsachen als Belege sein, damit alles soviel als möglich aktenmäßig würde. ${ }^{8}$

Seume grenzt seinen Späiergang als einen tatsachengetreuen (»geschichtsmäßig«), objektiven (»ohne Leidenschaft und Selbstsucht») und nachweisbaren (»aktenmäBig«) Bericht von den kindischen Erfindungen der Dichter ab: „Faktisch waren die Dinge so, wie ich sie erzähle «."

$\mathrm{Zu}$ den literarisch-fiktionalen Quellen journalistischer Wirklichkeitserzählungen gehört der realistische und naturalistische Roman des 19. Jahrhunderts, die Werke von Honoré de Balzac und Émile Zola in Frankreich, Theodor Fontane in Deutschland, Charles Dickens in England. Egon Erwin Kisch und Tom Wolfe etwa haben sich in ihren Konzeptionen des Journalismus ausdrücklich auf diese Autoren bezogen.

Zahlreiche bedeutende Schriftsteller haben sowohl im journalistischen als auch im fiktional-literarischen Modus erzählt. Dazu zählen im 18. Jahrhundert Daniel Defoe und Jonathan Swift, im 19. Jahrhundert, neben Zola, Fontane und Dickens, Heinrich Heine, Heinrich von Kleist und Mark Twain, im 20. Jahrhundert Joseph Roth, George Orwell, Jack London, Upton Sinclair, John Dos Passos, Ernest Hemingway, John Steinbeck, Gabriel García Márquez, Mario Vargas Llosa und viele andere mehr. ${ }^{10}$

Reportagen im heutigen Sinn zielen auf eine breite Öffentlichkeit, bemühen sich um Aktualität und Nachrichtenwert und setzen damit den Journalismus als soziales System in der funktional differenzierten Gesellschaft der Moderne voraus. Dieses System entstand im Laufe des 19. Jahrhunderts, als soziale und soziokulturelle Voraussetzungen (etwa die zunehmende Alphabetisierung), rechtliche Neuerungen (Aufhebung des staatlichen Anzeigenprivilegs) und technische Erfindungen in der Drucktechnik (Schnellpresse, Papiermaschine, Rotationsmaschine, Setzmaschine), in den Vertriebsmöglichkeiten (Eisenbahn) und in den Kommunikationsmedien (Telegraphie, Telefon) die Entstehung der Massenpresse ermöglichten. ${ }^{11}$ Die klassische Form der journalistischen Reportage wurde maßgeblich in den Jahren der Weimarer Republik durch Egon Erwin Kisch geprägt. Im Vorwort zu seiner Reportagensammlung Der rasende Reporter (1925) schreibt Kisch: »Der Reporter hat keine Tendenz, hat nichts zu rechtfertigen und hat keinen Standpunkt. Er hat unbefangen Zeuge zu sein und unbefangene Zeugenschaft zu liefern, so verläßlich, wie sich eine Aussage geben läßt«. ${ }^{12}$ Während

8 Johann Gottfried Seume: "Spaziergang nach Syrakus im Jahre 1802«, in: Ders.: Prosaschriften, Darmstadt 1974, S. 155-597, hicr: S. 158.

9 Seume $($ Inm. 8), S. 165.

10 Einc umfasscnde systematische und historische Untersuchung $\%$ den Wechselbexichungen zwischen Jiteratur und Journalismus in Luropa, Jateinamerika und den USA licfert Albert Chillón: Literatura y' periodismo. Una tradición de relaciones promisctas, Valencia 1999.

11 Vgl. Hans-Ulrich Wehler: Deutsche Gesellschaffsgeschichte 1815-1845/49, München 1987, S. 520-546 (»Die Ausdehnung des literarisch-öffentlichen Marktes und die Verdichtung der öffentlichen Kommunikation «).

12 Egon Erwin Kisch: Der rasende Reporter [1925], Gesammelte W'erke in Einzelansgaben, Bd. 6, 6. Aufl, Berlin/Weimar 1993, S. 9f. Vgl. Michael Geisler: Die literarische Reportage in Dentsch- 
Kisch hier im Gestus der Neuen Sachlichkeit vom Reporter vor allem »Hingabe an sein Objekt« und "Sachlichkeit« verlangte, um "die einfache Wahrheit» schreiben zu können, betonte er später, nach seinem Eintritt in die KPD im Jahr 1925, der Reporter solle eine engagierte Haltung einnehmen. "Gehäufte Fakten nur durch sich selbst wirken zu lassen«, »Tatsachen [...] einfach zu registrieren«, heißt es etwa in der Rede Reportage als Kunstform und Kampfform (1935), sei »phantasielos«. Der Reporter benötige eine »logische Phantasie«, um die Fakten zu einem »anklägerischen Kunstwerk " gestalten zu können. Aber auch in diesen späteren Jahren hielt Kisch am faktualen Geltungsanspruch des Reporters fest: »Bei aller Künstlerschaft muß er Wahrheit, nichts als Wahrheit geben, denn der Anspruch auf wissenschaftliche, überprüfbare Wahrheit ist es, was die Arbeit des Reporters so gefährlich macht, gefährlich nicht nur für die Nutznießer der Welt, sondern auch für ihn selbst, gefährlicher als die Arbeit des Dichters, der keine Desavouierung und kein Dementi zu fürchten braucht. "Obwohl die Reportage, als »Kunstform«, nach ästhetischen Kriterien konstruiert und zugleich, als »Kampfform«, politisch engagiert sein soll, habe sie vor allem »die Wahrheit präzis hinzustellen«. ${ }^{3}$

Auch Georg Lukács bestimmte die Gattung der Reportage durch ihren faktualen Geltungsanspruch. In seinem Aufsatz Reportage oder Gestaltung?, der 1932 in der Linkskurve, der Zeitschrift des Bundes der proletarisch-revolutionären Schriftsteller (BPRS) Deutschlands erschien, schreibt er: »In der Reportage kommt es nämlich vor allem darauf an, daß die angebenene Tatsachen in allen Details mit der Wirklichkeit übereinstimmen ${ }^{14}$ Allerdings habe die Reportage die Aufgabe, den dargestellten Einzelfall in einen übergeordneten Zusammenhang zu stellen und ihm dadurch allgemeinere Bedeutung zu geben; es gehe im journalistischen Erzählen um »die Verknüpfung der Tatsachen und ihrer Zusammenhänge, also auch des Besonderen und des Allgemeinen, des Individuellen und des Typischen, des Zufälligen und des Notwendigen ${ }^{15}$

\section{Journalistisches Erzählen zwischen Faktum und Fiktion}

Egon Erwin Kisch und Georg Lukács verstanden Reportagen als faktuale Texte. Dieser lange Zeit selbstverständliche, wahrheitsheischende Geltungsanspruch journalistischer Erzählungen wird seit den 1970 er Jahren im Zeichen dekonstruktivistischer und radikal-konstruktivistischer Auffassungen bestritten. Stattdessen wird, im Einzelnen mit ganz unterschiedlichen Argumenten, ein Panfiktionalis-

land. Möglichkeiten und Grenzen eines operativen Genres, Königstein 1982, und Ders.: „Berichterstattung in der Zcitung: Kommunikative und ästhetische Fragen«, in: Leonhard (Anm. 6), \$. $1712-1720$.

13 Ligon Firwin Kisch: "Reportage als Kunstform und Kampfform. Rede auf dem Internationalen Schriftstellerkongreß für die Verteidigung der Kultur in Paris im Juni 1935», in: Theodor Karst (Hg.): Reportagen, Stuttgart 1986, S. 163-166, hier: S. 165f. (sämtliche 'Zitate).

14 Georg Iukács: „Reportage oder Gestaltung?», in: Karst (Anm. 13), S. 159-163, hier: S. 162.

15 Lukács (Anm. 14), S. 159 
mus propagiert. So kommt - um ein Beispiel für viele zu nennen - Winfried Schulz in seiner wichtigen Studie über die Konstruktion von Realität in den Massenmedien zu dem Schluss, "daß das, was von den Autoren als Maßstab zur Beurteilung der Angemessenheit oder Ausgewogenheit, der Nachrichtenberichterstattung herangezogen wird, tatsächlich immer eine fiktive Realität ist«. ${ }^{16}$ Will man jedoch Formen und Funktionen des Erzählens im Journalismus angemessen und differenziert erfassen, muss man journalistisches und fiktional-literarisches Erzählen voneinander unterscheiden. Dafür ist es hilfreich, zwischen dem unvermeidlichen Konstruktionscharakter journalistischer Erzählungen (den sie mit allen anderen Formen und Feldern des Erzählens teilen) einerseits und ihrem angeblichen Fiktionscharakter andererseits zu trennen. ${ }^{17}$ Selbstverständlich prägen Faktoren wie die Selektion des verwendeten Informationsmaterials, die Verwendung bestimmter Erzähltechniken und rhetorisch-stilistische Gestaltungsformen maßgeblich die Nachrichten des Journalismus und das durch sie vermittelte Wirklichkeitsbild. Zweifellos konstruieren journalistische Nachrichten und Reportagen eine bestimmte Realität. Aber diese Texte sind eben auch auf eine intersubjektiv gegebene Wirklichkeit bezogen. Jeder narrative Text stellt etwas dar; doch unterscheiden sich faktuale Texte von fiktionalen dadurch, dass die in ihnen erzählte Wirklichkeit den Anspruch erhebt, auf konkrete Sachverhalte in unserer Wirklichkeit zu referieren. Es führt zu einer unnötigen begrifflichen Unschärfe, wenn man aus der unabweisbaren Tatsache der »Formung, Bildung und Gestalnung « journalistischer Texte ihren Fiktionscharakter ableitet. ${ }^{18}$

Ergiebiger ist es, die Verwendung genuin fiktionaler Erzählformen in faktualen Texten ernst zu nehmen, ohne deshalb gleich den Referentialitätsanspruch dieser Texte abzustreiten, der sie grundsätzlich von den Geschichten der fiktionalen Literatur unterscheidet. Sein faktualer Geltungsanspruch unterscheidet journalistisches Erzählen grundsätzlich von den fiktionalen Erzählungen der Literatur. Gerade durch diesen Anspruch kann es seine spezifische Funktion im System der Gesellschaft erfüllen, nämlich wahrheitsheischende Informationen zu liefern. In

16 Winfried 11. Schulz: Die Konstruktion von Realitat in den Massenmedien. Analyse der aktuellen Be richterstattung, 2. Aufl, Freiburg 1990, S. 27.

17 Zum hier verwendeten Fiktionsbegriff und grundsätzlich zur Unterscheidung zwischen faktualen und fiktionalen Erzähltexten vgl. die Einlcitung zu dics $\mathrm{cm}$ Band sowie Matías Martínce/Michacl Scheffel: Einfïbrung in die Erzähltheorie, 7. Aufl., München 2007, S. 9-20.

18 So z.B. Elisabeth Klaus: »enseits der Grenzen: Die problematische Unterscheidung zwischen Jakt und Fiktion«, in: Joan Kristin Bleicher/Bernhard Pörksen (Hg.): Grenzgänger. Formen des New Journalism, Wiesbaden 2004, S. 100-125, hier: S. 115: „Fakten sind [...] nur in ihrer kontextuellen Finbindung Kernbestandteil des Journalismus. Erst durch ihre fictiox, ihre Formung, Bildung und Gestaltung, werden sie zum journalistischen Produkt Finc differenzicrtere Auffassung formulicrt etwa Bernhard Pörksen: Konstruktivismus in der Journalistik »bedeutet nicht, dass man auch innerhalb der Jebenswelt und der eigenen Eirfahrungswirklichkeit auf (unvermeidlich temporäre und so\%ial verbindliche) Erkenntnissicherheiten verzichten muss. In der Sphäre der (journalistischen) Erfahrungswirklichkeit ist sclbstverständlich Konsensbildung möglich«. Bernhard l'örksen: "Journalismus als Wirklichkeitskonstruktion«, in: Martin Iöffelholz (Hg.): Theorien des Journalismus. Ein diskmsives Handbuch, 2., vollst. überarb. u. erw. Aufl., Wiesbaden 2004, S. 335-347, hier: S. $341 \mathrm{f}$. 
dem Moment, in dem journalistische Texte in mediale Zirkulation gebracht werden, gehen sie einen stillschweigenden Faktualitätspakt mit ihren Lesern ein.

Die Existenz eines solchen Paktes zeigt sich dann besonders deutlich, wenn er verletzt wird. $\mathrm{Zu}$ den größten Skandalen des investigativen Journalismus in den Printmedien der letzten Jahrzehnte gehört Janet Cookes 1980 in der W ashington Post publizierte, eindrucksvolle Reportage Jimmy's World über die trostlose Existenz eines achtjährigen, heroinabhängigen Jungen in der Hauptstadt der USA. ${ }^{19}$ Die Reportage löste eine zweiwöchige erfolglose Suche der städtischen Behörden nach Jimmy aus und wurde mit dem Pulitzer Preis ausgezeichnet. Später stellte sich heraus, dass Cooke Jimmy und seine traurige Lebensgeschichte erfunden hatte. Ähnlich großes Aufsehen erregte der Journalist Jayson Blair. Er wurde 2003 von der New York. Times, Inbegriff des faktentreuen Journalismus, entlassen, nachdem bekannt geworden war, dass Blair in dieser Zeitung jahrelang Hunderte von teils frei erfundenen, teils plagiierten Berichten publiziert hatte.

Die empörten Reaktionen auf solche Fälschungen machen deutlich: Journalistische Texte werden als faktuale Erzählungen verstanden, die beanspruchen, dass das, was sie erzählen, wahr ist. Die Wahrheit, um die es dabei geht, liegt in der nachprüfbaren Referenz auf konksete Tatsachen, d.h. auf einzelne, räumlich und zeitlich bestimmte Sachverhalte und Ereignisse unserer Wirklichkeit. Es hat Janet Cooke nichts genützt, dass sie zu ihrer Verteidigung vorbrachte, der von ihr beschriebene Jimmy existiere zwar nicht, dafür aber zahllose reale Kinder, die in der Figur des Jimmy exemplarisch dargestellt würden. Welchen Anspruch journalistische Reportagen ansonsten auch immer erheben: sie unterstehen jedenfalls der Erwartung, im konkreten Sinn wahr zu sein.

\section{Drei Fallanalysen}

\section{Günter Wallraff}

Günter Wallraffs Rollenreportagen setzen seit den 1960er Jahren die Tradition der klassischen Reportage der Zwanziger Jahre fort, insofern sie dic Erfahrung konkreter sozialer Wirklichkeit vermitteln möchten. Egon Erwin Kisch hatte, wie erwähnt, vom Reporter "unbefangene Zeugenschaft" verlangt. ${ }^{20}$ Mehr noch als auf Kisch beruft sich Wallraff auf den russischen Schriftsteller Sergej Tretjakow: "Er entwarf das Bild eines Autors, der vom Beobachter zum operierenden Schriftsteller wird, der sich in die Prozesse der Veränderung hineinstellt«. ${ }^{21}$ Wallraff radikalisiert die Haltung des Reporters als teilnehmender Beobachter, indem er sich selbst intensiv den Missständen aussetzt, über die er berichtet. In dem

19 http://www.uncp.edu/home/canada/work/markport/lit/litjour/spg2002/cookc.htm, Aufruf 21.3.09.

20 Kisch (Anm. 12), S. 9f.

21 Günter Wallraff: mSicht von untenc. Interview mit Alfred Fichhorn«, Neute Deutscbe Literatur 24 (1976), II. 8, S. 147-149, hier: S. 148f. 'Zu Wallraffs Rollenrcportagen s. Ulla Itahn/ Michael Töteberg: Günter W'allraff, München 1979. 
Artikel Kisch und ich heute. Über die logische Phantasie (1977) schreibt er: »Ich muß selbst erst zum Betroffenen, notfalls zum Opfer werden, um über die Situation der Opfer dieser Gesellschaft schreiben zu können«. ${ }^{22}$ Nicht schon die sympathisierende Beobachtung sozialer Opfer, sondern erst das Erlebnis ihrer Situation am eigenen Leib ermöglichen, so Wallraff, eine authentische Berichterstattung. Deshalb hat er paradoxerweise immer wieder falsche Identitäten angenommen: Durch verdeckte Identitäten möchte er Wahrheit aufdecken. Mehr noch, mit absichtlichen Provokationen verändert er aktiv in seinen Rollenspielen gegebene Situationen und treibt soziale Missstände auf die Spitze. Auf diese Weise hat er seit seinen ersten Reportagen aus der industriellen Arbeitswelt (Industriereportagen. Als Arbeiter in deutschen Großbetrieben, 1970; zuerst 1966 erschienen unter dem Titel Wir brauchen dich - Als Arbeiter in deutschen Industriebetrieben) zahllose und unterschiedlichste Milieus kennengelernt und dargestellt. Zu seinen bekanntesten Arbeiten gehören Der Aufmacher. Der Mann, der bei Bild Hans Esser war (1977) über seine verdeckte Tätigkeit als Journalist in der Hannoveraner Redaktion der BildZeitung und sein erfolgreichstes Buch, Gan₹ unten. (1985). Walltraff berichtet hier über seine Erlebnisse als angeblicher Türke Ali Levent z.B. in der Küche von McDonalds-Restaurants, als Leiharbeiter auf Großbaustellen, in Industrieanlagen von Thyssen oder bei Medikamentenversuchen. Im Text erscheint durchgängig die Sprecherinstanz wich (Ali)«, während das Vorwort (»Die Verwandlung«) mit "Günter Wallraffu unterzeichnet ist. In diesem Vorwort schreibt Wallraff: „Sicher, ich war nicht wirklich ein Türke. Aber man muß sich verkleiden, um die Gesellschaft zu demaskieren, muß täuschen und sich verstellen, um die Wahrheit herauszufinden «. ${ }^{23} \mathrm{Nun}$ ist aber die Sprecherinstanz »ich (Ali) « keineswegs zu verwechseln mit fiktiven Erzählern in fiktional-literarischen Texten. Während der Ich-Erzähler Oskar Marzerath in der Blechtrommel die unwahrscheinlichsten Dinge behaupten kann, ohne dass wir seinen Autor Günter Grass der Lüge bezichtigen, erhebt wich (Ali)« einen faktualen Geltungsanspruch: So ist es gewesen, sso habe ich es erlebt<. Dieser Anspruch wird selbstverständlich nicht außer Kraft gesetzt durch Wallraffs Hinweis, er habe wzum Schutz meiner Kollegen ihre Namen in diesem Buch zum großen Teil verändern« müssen. ${ }^{24}$ Gerade die vermutete Gefahr einer arbeitsrechtlichen oder strafrechtlichen Verfolgung unterstreicht den referentiellen Charakter des Textes.

Soweit zu Wallraffs Erneuerung und Radikalisierung des klassischen, wahrheitsheischenden Reportage-Journalismus. Es gibt aber auch Autoren, die den faktualen Geltungsanspruch journalistischen Erzählens herausfordern, indem sie mit der Grenze zwischen fiktionalem und faktualem Erzählen spielen. Betrachten wir zwei dieser Grenzgänger.

22 Günter Wallraff: »Kisch und ich heute. Über die logische Phantasie«, Die Zeit, 11.11.1977. Wallraff stcllt sich hicr in die Tradition Kischs, fügt aber hinzu, er habe dessen Reportagen erst spät kennengelernt.

23 Günter Wallraff: Ganz unten, Köln 1985, S. 12.

24 Wallraff (Anm. 23), S. 13. 
New Joumalism

1965 erschien Truman Capotes In Cold Blood. Das Buch trägt den Untertitel »A True Account of a Multiple Murder and Its Consequences« und erzählt die Geschichte der Ermordung der vierköpfigen Familie Clutter im Jahr 1959 nahe der Kleinstadt Holcomb in Kansas (USA). In Cold Blood enthält eine Reihe von Passagen, die streng genommen den übermenschlichen Standpunkt eines allwissenden Erzählers voraussetzen, beispielsweise Dialoge zwischen verschiedenen Protagonisten, die in direkter Rede zitiert werden, obwohl sie nicht aufgezeichnet wurden, oder auch die Wiedergabe von Gedanken und Gefühlen der Protagonisten in erlebter Rede oder mit Hilfe anderer Techniken der Bewußtseinsdarstellung, die eigentlich einen direkten Zugang zum Bewußtsein dieser Personen voraussetzen. Ungeachtet dieser Fiktionalisierungen beansprucht Capote jedoch in den Paratexten von In Cold Blood faktuale Wahrheit. Das zeigt sich außer in dem erwähnten Untertitel mit der Kennzeichnung strue account « auch in den »Acknowledgments«, die dem Hauptext vorangestellt sind. Auf der Schwelle zur eigentlichen Erzählung wird dem Leser hier mitgeteilt, dass Capotes Geschichte vollständig durch Recherchen belegt sei: »All the material in this book not derived from my own observation is either taken from official records or is the result of interviews with the persons directly concerned, more often than not numerous interviews conducted over a considerable period of time ${ }^{25}$ Die fiktionalisierenden Erzählverfahren sind begründungsbedürftige Lizenzen eines Textes, der gleichwohl einen faktualen Geltungsanspruch erhebt. Deshalb bezeichnete Capote sein Buch als »non-fiction novel ${ }^{26}$ Die Behauptungen, die in fiktionalen Texten erhoben werden, können nicht dem realen Autor (z.B. Günter Grass), sondern nur einem fiktiven Erzähler (z.B. Oskar Matzerath in Grass' Roman Die Blechtronmel) zugerechnet werden - sonst müssten wir Grass der Lüge bezichtigen, weil er uns wider besseres Wissen von Figuren und Ereignissen erzählt, die gar nicht exisitiert haben. Capote aber muss für die Wahrheit (oder zumindest für die Plausibilität) seiner Darstellung geradestehen. Anders als in Texten der fiktionalen Literatur mit rechten< allwissenden Erzählern, deren Behauptungen vom Leser ohne weiteres als wahr (wwahr allerdings nur in der fiktiven Welt des Romans) hingenommen werden, muss Capote die fiktionalisierenden Passagen als zwar hypothetische, aber aufgrund umfangreicher Recherchen zumindest plausible Darstellung eines vermutlich realen Sachverhalts rechtfertigen. Ungeachtet aller literarischen< Erzähltechniken spricht er stets in eigener $\mathrm{Sa}$ che. Nur so ist es z.B. überhaupt möglich, dass man In Cold Blood gelegentlich eine verfälschende Darstellung der vier Morde in Kansas vorgeworfen hat. Dage-

25 Truman Capote: In Cold Blood. A True Account of a Multiple Murder and Its Conseqnences, New York 1965, o.s. '/u Capotes Erzähltechnik s. John Itollowell: "Truman Capote's Nonfiction Novelu, in: Ders.: Fact \& Fiction. The New Jorrnalism and the Nonfiction Novel, Chapel Hill 1977, S. 63-86.

26 George Plimpton: "Truman Capote: An Intervicw《 [1966], in: Ronald Weber (IJg): The Reporter as Artist: A Look at The New Journalisw Controversy, New York 1974, S. 188-206, hier: S. 189 
gen wäre es sinnlos, die Behauptungen des fiktiven Erzählers eines Romans als falsch zurückzuweisen.

Capotes Schreibweise, die am faktualen Geltungsanspruch journalistischen Erzählens festhält, aber zugleich die dafür üblichen Darstellungskonventionen durchbricht, ist für eine Gruppe von US-amerikanischen Journalisten der 1960er und 1970er Jahre charakteristisch, die unter dem Begriff New Joumalism zusammengefasst werden. Zu ihr gehören neben Capote u.a. Gay Talese, Norman Mailer, Hunter Thompson und Tom Wolfe. ${ }^{27}$ Die Reportagen des New Journalism zeigen, dass die Opposition zwischen fiktionaler und faktualer Rede nicht trennscharf ist, sondern Kombinationen in unterschiedlicher Art und Weise zulässt. Unterscheidet man zwischen der Fiktivität der erzählten Geschichte (besitzen die erzählten Sachverhalte eine Referenz in unserer Wirklichkeit oder nicht?) und der Fiktionalität der Erzählrede (wahrheitsheischende Rede des realen Autors oder imaginäre Rede eines fiktiven Erzählers?), dann lassen sich die Reportagen des New Journalism als faktuale Erzählungen mit teilweise fiktionalisierenden Erzählverfahren charakterisieren. ${ }^{28}$

\section{Tons Kunmer}

Der Schweizer Journalist Tom Kummer veröffentlichte zwischen 1996 und 1999 vor allem im Magazin der Süddeutschen Zeitung, aber auch im ZEIT Magarin und in weiteren Zeitungen Dutzende von Interviews mit Hollywood-Stars und anderen Berühmtheiten der US-amerikanischen Populärkultur. Im Jahr 2000 stellte sich heraus, dass die meisten dieser Interviews zu großen Teilen oder ganz erfunden worden waren. Kummer war den Stars zumeist gar nicht persönlich begegnet. Die Aufdeckung der Fälschung führte zur Beendigung der Zusammenarbeit der betroffenen Redaktionen mit Kummer und zur Entlassung der verantwortlichen Redakteure, aber auch, anders als in den Fällen von Janet Cooke und Jayson Blair, zu hitzigen Debatten über die Legitimität solcher Interviews. Kummer hat nämlich seinen $>$ Borderline-Journalismus mit interessanten Argumenten verteidigt. Es gehe ihm um eine "Neudefinition von Realität im Journalismus « ${ }^{29}$ Er habe in seinen Texten - Kummer nennt sie »konzeptionelle Interviews $\aleph^{30}$ - die Stars nicht als private Personen, sondern als Teil der Imageindustrie Hollywoods porträtieren wollen. Deswegen handele es sich nicht um Fälschungen, da seine Interviews wahrheitsgetreu das Image der Stars vorstellten. Nun geben die Interviews keine expliziten Hinweise auf ihren fiktiven Status. Ein unwissender Leser muss sie für

27 Vgl. Tom Wolfc/Edward Warren Johnson (Hg.): The New Journalism. With an Anthology, New York 1973.

28 Zu den Begriffen ,faktuak, ,fiktional und sfiktive vgl. dic Einleitung zu diesem Band.

29 Tom Kummer: »Manifesto. Die Matrix der Wirklichkeitsentwürfe«, in: http://tomkum mer.be/manifesto, Aufruf 14.5.05; diese Internetscitc ist inzwischen nicht mchr zugäng. lich. Zum Fall Kummer s. Holger Schulze: „Wirklichkcit messen. Tom Kummer vs. Reality«, in: http://nachdemfilm.de/no2/sul01dts.html, Aufruf 20.4.09. Kummer selbst hat sich dagegen gewehrt, seine Star-Intervicws als >Borderline-Journalismus $\%$ u bezeichnen.

30 Tom Kummer: $m$ Wow, das ist gutcr Stoff. Intervicw mit Martin Sutcre, Tagesspiegel, 02.12.2001 
authentisch halten. Allerdings spielen sie hier und da auf Kummers Konzeption eines zeitgemäßen Journalismus an. Im angeblichen Gespräch mit Courtney Love sagt diese: „Ich betrüge so echt, ich bin jenseits von Betrugu. Jenny McCarthy bittet ihren Interviewpartner, sie »Jenny, deine beste TV-Freundin« zu nennen, und behauptet, »daß sich die Menschen über Images eine Meinung bilden und daß man diese Tatsache nutzen sollte«. ${ }^{31}$ Der Rapper Snoop Doggy Dogg antwortet auf die Frage Kummers "Dann ist also alles, was Sie mit Ihrer Stimme verbreiten, bloß Dichtung? «: „Was denn sonst. Mich interessiert nicht die Wahrheit, ich inszeniere meine Wahrheit«. ${ }^{32}$ Und bei Pamela Anderson kommt Kum-

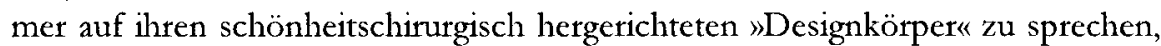
der sie zur »Ikone des Körperkults« und damit zu einem »künstlichen Menschen« werden ließ: „Pamela, eigentlich verkörperst du ja den materialisierten Zeitgeist annähernd perfekt: Die Wirklichkeit verliert sich in der Simulation, und der Körperkult ist die konsequenteste Reaktion darauf « ${ }^{33}$ In einem »Manifesto« erläuterte Kummer nachträglich seine Auffassung von Journalismus:

Es ist wirklich nicht mchr leicht, Journalist $z u$ sein. Fine Armada von Authentizssten klebt einem im Nacken und fordert die Wahrheit, die einzige wahrhaftige Wirklichkeit. [... W W sind in die vom Schein beherrschte Welt der Postmoderne hincingeboren worden, deren bestimmendes Element dic Show ist. In der Show gibt es keine Wahrheit, sondern Fiffekte. [... Meine journalistische Erfahrung war immer die, dass mit dem Auftauchen von Journalisten dic Wirklichkcit implodiert. Ich habe das immer wieder gesehen, ob in der Wartestellung auf den ersten Golfkrieg im Intercontinental-Hotel von Amman, wo die Wirklichkeit von isolierten Journalisten choreographicrt wurde. [...] Objektivität ist genauso wie Wahrheit und Wirklichkeit in den Medien ein reiner Mythos. ${ }^{34}$

Kummer beruft sich hier auf Jean Baudrillards Auffassung, unsere postmoderne Welt der Simulakren und Simulationen setze den Begriff der Wirklichkeit außer Kraft: wsimulation threatens the difference between strues and ifalses, between reak and simaginary «. $35 \mathrm{Ob}$ das eine plausible These ist oder nicht, braucht hier nicht diskutiert zu werden. Die öffentlichen Reaktionen auf die Enthüllung des fingierten Charakters von Kummers Star-Interviews zeigen jedenfalls, dass es für den Umgang des Publikums mit journalistischen Texten nach wie vor einen entscheidenden Unterschied ausmacht, ob es sie als fiktional oder als faktual versteht. Kummers Texte wurden eben nicht als wahrheitsneutrale Simulationen, sondern als Fälschungen authentischer Interviews angesehen - und ihr Autor und die übrigen Verantwortlichen entsprechend bestraft.

31 Gibt es etwas Stärkeres als V'etfiubnnng, Miss Stone? Star-Interviews von Tom Kunmer, München 1997, S. 101, 176, 177. Es handelt sich hicr zwar nicht um narrative Texte, sondern um Intervicws; für das Vcrständnis des faktualen Geltungsanspruchs journalistischen Erzählens ist Kummers Position dennoch aufschlussreich.

32 Gibt es etwas Stärkeres als L'erfibrung, Miss Stone? (Anm. 31), S. 72.

33 Gibt es etwas Stärkeres als Lerfibrung, Miss Stone? (Anm. 31), S. 27.

34 Kummer (Anm. 29).

35 Jcan Baudrillard: "Simulacra and Simulations«, in: Ders.: Selected Writings, Stanford 1988, S. 166-184, hicr: S. 168. 
Wie wir gesehen haben, fordern die Reportagen des New Journalism und Tom Kummers Interviews den faktualen Geltungsanspruch journalistischer Wirklichkeitserzählungen heraus. Die grundsätzliche Existenz dieses Anspruchs bestätigen sie aber eher, als dass sie ihn beseitigen. Durch ihr Spiel mit den Grenzen zwischen faktualem und fiktionalem Erzählen und zwischen Faktum und Fiktion zwingen sie allerdings zu einer genaueren Beschreibung des Geltungsanspruchs journalistischen Erzählens.

\section{Forschungsgeschichte}

Die Erforschung journalistischen Schreibens als ein besonderer Modus des Erzählens erlebte einen ersten Höhepunkt in den 1920er Jahren in Beiträgen von Egon Erwin Kisch, Georg Lukács, Kurt Tucholsky u.a. Insbesondere verglichen sie die Leistungen literarischen und journalistischen Erzählens und diskutierten den spezifischen Wahrheitsanspruch von Reportagen. Praxisbezogene Handbücher zum journalistischen Schreiben entstanden zuerst in den Jahren zwischen den Weltkriegen in den USA. Eine bis heute anhaltende Diskussion über die Legitimität fiktionalisierender Darstellungstechniken in journalistischen Texten setzte Mitte der 1960er Jahre mit dem Aufkommen des New Journalism ein. Tom Wolfe lieferte mit seinem Aufsatz "The New Journalism" (1973) die bekannteste Selbstbeschreibung dieser Richtung. ${ }^{36}$ Während die Vertreter des New Journalism ungeachtet ihrer Verwendung literarischer Erzähltechniken am faktualen Geltungsanspruch ihrer Reportagen festhalten, wird seit den 1970er Jahren unter dem Einfluss von postmodemen Auffassungen einerseits (hier war Jean Baudrillards Theorie des Simulakrums besonders populär) und von manchen radikal-konstruktivistischen Theorien andererseits (in der Nachfolge von Niklas Luhmanns Systemtheorie) die Berechtigung der Unterscheidung zwischen fiktional-literarischem und faktual-journalistischem Erzählen bestritten. Seit einigen Jahren scheint die Überzeugungskraft dieser Positionen nachzulassen. Hingegen hält der Einfluss des New Journalism an. Auf ihn berufen sich aktuelle Ansätze eines ,Narrative Journalism (auch: ,Literary Journalism<, Immersive Journalism). Das 2001 an der Harvard University gegründete Nieman Program on Narrative Journalism beispielsweise ist ausdrücklich den erzählenden Aspekten des Journalismus gewidmet. ${ }^{37}$ In der Tradition des New Journalism wird dort ein journalistisches Schreiben gefordert, das literarische Erzähltechniken wie szenisches Erzählen, komplexe Figurendarstellung, dramatische Handlungsführung und psychologisierende (intern fokalisierte) Erzählstandpunkte verwendet. Die rasch zunehmende Bedeutung des Internets für die Verbreitung aktueller Nachrichten könnte dazu führen, dass im Printjournalismus der Aktualitätsgrad der Meldungen unwichtiger wird und man sich dort stattdessen mehr auf umfangreichere Berichte und Re-

36 Tom Wolfe: "The New Journalism«, in: Ders./Warren (Anm. 27), S. 1-52. Auch die in diesem Band enthaltene Auswahl von Beispieltexten bestimmte maßgeblich das Bild des New Journalism.

37 http://www.nieman.harvard.edu/narrative/home.aspx, Aufruf 20.3.09. 
portagen konzentriert. Die Bedeutung des Erzählens und die Vielfalt der Erzählformen würden dann wohl eher $z u-$ als abnehmen.

\section{Kommentierte Auswahlbibliographie}

Bleicher, Joan Kristin/Pörksen, Bernhard (Hg): Grenżänger. Formen des New Journalism, Wiesbaden 2004. - Die Aufsätze untersuchen literarische und fiktionalisierende Formen des erzählenden Journalismus seit dem New Journalism bis hin zu Texten Tom Kummers, der Popliteratur und der Zeitschrift Tempo, sowie entsprechende Phänomene im Fernsehen; einige Beiträge diskutieren genereller den Objektivitätsanspruch journalistischer Texte.

Geisler, Michael: Die literarische Reportage in Deutschland. Möglichkeiten und Greñen eines operativen Genres, Königstein 1982. - Geisler gibt einen historischen Überblick über Theorien und Formen der literarischen Reportage im 20. Jahrhundert.

Haller, Michael: Die Reportage, 6., überarb. Aufl., Konstanz 2008. - Standardwerk über journalistische Darstellungs- und Erzähltechniken für den praktischen Gebrauch.

Löffelholz, Martin (Hg.): Theorien des Joumalismus. Ein diskursives Handbuch, 2., vollst. überarb. u. erw. Aufl., Wiesbaden 2004. - Der Band enthält Überblicksdarstellungen über aktuelle Theorien des Journalismus mit einem Schwerpunkt auf Ansätzen der Systemtheorie, der Kommunikationstheorie und der Cultural Studies.

Weischenberg, Siegfried: Nachrichten-Joumalismus. Anleitungen und Qualitätsstandards für die Medienpraxis, Wiesbaden 2001. - Standardwerk über journalistische Darstellungs- und Erzähltechniken für den praktischen Gebrauch. 
Christian Klein/Matías Martínez (Hrsg.)

\section{Wirklichkeitserzählungen}

Felder, Formen und Funktionen

nicht-literarischen Erzählens

2009

Verlag J. B. Metzler Stuttgart · Weimar 